\title{
Absence of the zero bias peak in vortex tunneling spectra of high temperature superconductors
}

\author{
Congjun $\mathrm{Wu}^{1,2}$, Tao Xiang ${ }^{2}$ and Zhao-Bin $\mathrm{Su}^{2}$ \\ ${ }_{1,2}$ Department of Physics, Peking University, Beijing 100087, The People's Republic of China \\ ${ }^{2}$ Institute of Theoretical Physics, Chinese Academy of Sciences, P.O.Box 2735, Beijing 100080, The People's Republic of \\ China
}

\begin{abstract}
The $c$-axis tunneling matrix of high- $T_{c}$ superconductors is shown to depend strongly on the inplane momentum of electrons and vanish along the four nodal lines of the $d_{x^{2}-y^{2}}$-wave energy gap. This anisotropic tunneling matrix suppresses completely the contribution of the most extended quasiparticles in the vortex core to the $c$-axis tunneling current and leads to a spectrum similar to that of a nodeless superconductor. Our results give a natural explanation of the absence of the zero bias peak as well as other features observed in the vortex tunneling spectra of high- $T_{c}$ cuprates.
\end{abstract}

Recently there has been intensified attention on the electronic structure in the $d_{x^{2}-y^{2}}$-wave vortex state of high- $\mathrm{T}_{c}$ superconductors [1] 13]. From the solution of the Bogoliubov-de Gennes (BdG) equation, many authors [5.8.9] showed that in a $d$-wave superconductor there are no vortex core bound states and the local density of states (LDOS) at the core center has a broad zero energy peak. However, in contrast to the theoretical prediction, the scanning tunneling spectrum (STS), which is generally assumed to be proportional to the LDOS, at the center of a vortex core in both $\mathrm{YBa}_{2} \mathrm{Cu}_{3} \mathrm{O}_{7-\delta}$ (YBCO) [1] and $\mathrm{Bi}_{2} \mathrm{Sr}_{2} \mathrm{CaCu}_{2} \mathrm{O}_{8+\delta}$ (BSCCO) [2,3] have revealed a number of features which are totally unexpected in a pure d-wave superconductor: first, there is zero peak at zero bias; second, the coherent peaks at the superconducting gap edges are largely suppressed; and third, as in a $s$-wave superconductor, localized vortex core states seem to exist at $\pm 5.5 \mathrm{meV}$ in $\mathrm{YBCO}$ [1] and $\pm 7 \mathrm{meV}$ in $\mathrm{BSCCO}$ [3].

Many theoretical concepts, including spin-charge separation, $\mathrm{SO}(5)$ symmetry, and time reversal symmetry breaking pairing states have been invoked to resolve this discrepancy between theories and experiments [7,10, 12,13]. A simple theoretical explanation for the vanishing zero bias peak is that the pairing state inside the core has $d_{x^{2}-y^{2}}+i d_{x y}$ or $d_{x^{2}-y^{2}}+i s$ wave symmetry and therefore as in a $s$-wave superconductor vortex bound states exist in these materials [8]. However, there is no direct experimental evidence for the existence of such time-reversal symmetry broken states. In the $\mathrm{SO}(5)$ theory the vortex core can be either metallic or insulating depending on the doping level or other physical parameters of a high-Tc material [7] 10]. This is consistent with the STS data of YBCO [11] and BSCCO [2,3]. Under the framework of the U(1) gauge theory, Franz et al 12] argued that the pseudogap behavior of the STS at the core center is a result of the charge-spin separation and what the experiments found is the excitation spectrum of paired spinons which are not affected by external fields.

Nearly all theoretical analyses of the STS at the vortex core have assumed that the tunneling matrix element is a momentum independent constant. Under this assump- tion it can be shown that the c-axis tunneling differential conductance measured in the STS experiments is proportional to the LDOS of low lying excitations. However, for high- $T_{c}$ cuprates, the c-axis hopping integral of electrons is highly anisotropic 14,16 and depends strongly on the in-plane momentum. In particular, it has the same nodal structure as the $d_{x^{2}-y^{2}}$-wave gap function in tetragonal cuprates like BSCCO. The tunneling matrix is proportional to the c-axis velocity of electrons, thus it should also depend strongly on the in-plane momentum of electrons. This anisotropic c-axis hopping integral has strong impact on the c-axis transport of quasiparticles in the superconducting state. It leads to some peculiar temperature dependence of the $c$-axis penetration depth [15, 17 as well as microwave conductivity [18] at low temperatures and suppresses significantly the c-axis tunneling conductance [19].

In this paper, we present a theoretical analysis of the STS in the vortex core of high- $T_{c}$ superconductors. We shall show that the c-axis STS is dramatically modified by the anisotropy of the tunneling matrix. Our work provides a natural explanation for the absence of the zero bias peak in the STS at the core center. It shows that the pseudogap feature of the tunneling conductance at the core center is mainly due to the suppression of the tunneling current of low energy quasiparticles by the anisotropic c-axis hopping integral. A critical test for our theory is to measure the tunneling conductance in a vortex core which is parallel to the $\mathrm{CuO}_{2}$ planes. The tunneling matrix parallel to the $\mathrm{CuO}_{2}$ planes is not sensitive to the pairing symmetry and thus can be taken approximately as a constant. In this case, a broad zero bias peak is expected to exist in the STS of the vortex. This anisotropic feature of the STS is absent in other theories.

To study the structure of a $d$-wave vortex along the $c$-axis, we have performed a self-consistent calculation of the Bogoliubov-de Gennes ( $\mathrm{BdG}$ ) equation on a square lattice. Before presenting the detailed numerical results, let us briefly discuss why there is a broad zero-energy

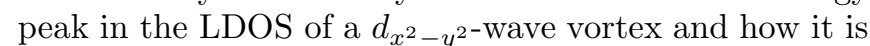
suppressed in the scanning tunneling experiment by the anisotropic $c$-axis hopping integral. 
For conventional s-wave superconductors, it was established many decades ago 20 that discrete quasiparticle states with a characteristic excitation energy given by $\Delta^{2} / 2 \varepsilon_{F}$ exist in a vortex core, where $\Delta$ is the bulk gap and $\varepsilon_{F}$ is the Fermi energy. Intuitively, this can be understood by drawing an analogy to a simple quantum mechanical problem of a particle in a cylindrical well of radius $\xi \sim v_{F} / \pi \Delta$ and height $\Delta$. However, in a $d_{x^{2}-y^{2-}}$ wave superconductor, the radius and height of the analogous potential well depends on the polar angle on the Fermi surface. Along the four node directions, $\Delta$ vanishes and $\xi$ diverges. In this case the quasiparticle is extended along the node directions and there is no truly localized core states. Thus unlike the s-wave case, the

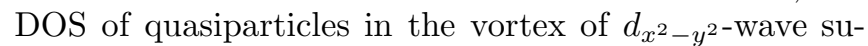
perconductors is finite even at zero energy. From the numerical solution of the $\mathrm{BdG}$ equation for a $d_{x^{2}-y^{2} \text {-wave }}$ superconductor, it has been further shown that there is a broad peak at zero energy in the LDOS of the vortex core. This peak mainly arises from the quasiparticle excitations along the four node lines.

The STS is determined by the LDOS and the tunneling matrix elements. In high- $T_{c}$ materials, since the $c$-axis tunneling matrix vanishes along the four node lines, the contribution of the most extended quasiparticles, around the gap nodes, to the tunneling current is completely suppressed. This is why the zero energy peak in the LDOS is absent in the STS of the vortex.

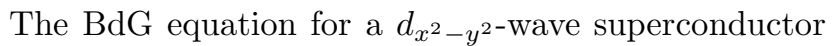
on a lattice is given by 21]

$$
\left(\begin{array}{ll}
\widehat{H}_{0} & -\widehat{\Delta} \\
-\widehat{\Delta}^{*} & -\widehat{H}_{0}^{*}
\end{array}\right)\left(\begin{array}{l}
u_{n}(i) \\
v_{n}(i)
\end{array}\right)=E_{n}\left(\begin{array}{l}
u_{n}(i) \\
v_{n}(i)
\end{array}\right)
$$

and

$$
\begin{aligned}
\widehat{H}_{0} u_{n}(i) & =-t \sum_{\delta} u_{n}(i+\delta)-\mu u_{n}(i), \\
\widehat{\Delta} v_{n}(i) & =\sum_{\delta} \Delta_{i, \delta} v_{n}(i+\delta),
\end{aligned}
$$

where $\delta$ denotes a nearest-neighbor vector, $t$ is the hopping constant, and $\mu$ is the chemical potential. $\left(u_{n}(i), v_{n}(i)\right)$ is the wave function of Bogoliubov quasiparticles. We have ignored the coupling to the vector potential $A$ in the hopping term in the limit of an extremely type II superconductor. The effect of the gauge field is manifested in the winding number of the order parameter in the calculation. The self-consistent condition for the gap parameter is

$$
\Delta_{i \delta}=\frac{g}{2} \sum_{n}\left[u_{n}(i) v_{n}^{*}(i+\delta)+u_{n}(i+\delta) v_{n}^{*}(i)\right] \tanh \frac{\beta E_{n}}{2}
$$

where $g$ is the pair coupling constant.

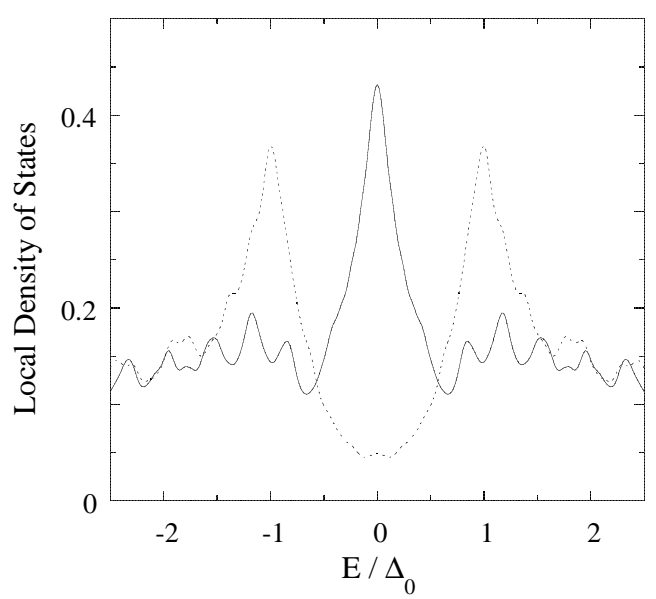

FIG. 1. Local density of states at the center of the vortex at site $(0,0)$ (solid line) and at site $(10,10)$ (dotted line) on a $51 \times 51$. The small oscillations of the curves are due to the finite size effects. The energy is normalized by the maximum energy gap $\Delta_{0}=0.6 t$. The broadening parameter is $\gamma=0.05 t$.

The LDOS is determined by the wavefunctions of quasiparticles:

$$
\begin{aligned}
\rho(i, E) & =\sum_{n} \rho(i, n, E) \\
\rho(i, n, E) & =\left|u_{n}(i)\right|^{2} L\left(E-E_{n}\right)+\left|v_{n}(i)\right|^{2} L\left(E+E_{n}\right),
\end{aligned}
$$

where $L(x)=\gamma / \pi\left(x^{2}+\gamma^{2}\right)$ and $\gamma$ an energy broadening parameter. $\rho(i, E)$ is a sum of the LDOS of the $n^{\prime}$ th vortex eigenstate at site $i, \rho(i, n, E)$. Figure 1 shows the LDOS at site $(0,0)$ (core center) and site $(10,10)$ on a $51 \times 51$ square lattice with open boundary conditions. The parameters used are $g=1.5$ and $\mu=0$. In agreement with other numerical results [5, 8 , we find that the LDOS at the core center exhibits a broad peak at $\omega \sim 0$. This peak drops very quickly with the distance from the core center. The LDOS at site $(10,10)$ behaves in nearly the same was as in an homogeneous $d_{x^{2}-y^{2}}$-wave superconductor. There is a coherent peak at the gap edge $\left(|\omega| \sim \Delta_{0}\right)$ at $(10,10)$. However, at the core center, this coherent peak is suppressed.

The tunneling current between a normal tip and a superconductor at a bias voltage $V$ is given by

$$
\begin{aligned}
I(i, V)= & 2 e \sum_{n, p}\left|T_{n k_{z}, p}\right|^{2} \int_{-\infty}^{+\infty} \frac{d \varepsilon}{2 \pi} A_{S}(n, i, \varepsilon) \\
& A_{N}(p, \varepsilon+e V)\left[n_{F}(\varepsilon)-n_{F}(\varepsilon+e V)\right]
\end{aligned}
$$

where $A_{S}(n, i, \varepsilon)=2 \pi \rho(i, n, \varepsilon)$ and $A_{N}(p, \varepsilon)=2 \pi \delta(\varepsilon-$ $\left.\xi_{p}\right)$ are the spectral functions of the superconductor and normal tip, respectively. $p$ is the momentum of electrons in the normal tip and $\left(n, k_{z}\right)$ are the quantum numbers of the vortex states. The tunneling matrix element $T_{n k_{z}, p}$ is proportional to the velocities of electrons along the tunneling direction on both sides of the junction [22]:

$$
\left|T_{k_{z} n ; p}\right|^{2}=D\left(\varepsilon_{z}\right)\left|v_{p}^{n} v_{k_{z}, n}^{s}\right|
$$


where $D\left(\varepsilon_{z}\right)$ is the barrier transmission coefficient. $v_{p}^{n}$ is the Fermi velocity in the normal tip, which can be approximately taken as a constant. The velocity of the $n^{\prime}$ th vortex state $v_{k_{z}, n}^{s}$ is determined by the energy dispersion of quasiparticles along the $c$-axis. To the lowest order approximation of $t \perp$ (defined below), it is proportional to the expectation value of the $c$-axis current operator in the $n^{\prime}$ th vortex state.

For tetragonal high- $T_{c}$ compounds, the $c$-axis energy dispersion of electrons has been shown to have the form

$$
\varepsilon_{z}(k)=-t_{\perp}\left(\cos k_{x}-\cos k_{y}\right)^{2} \cos k_{z},
$$

where $t_{\perp}$ is the $c$-axis hopping constant. This anisotropic $c$-axis hopping integral results from the hybridization between antibonding $O 2 p$ and unoccupied $C u 4 s$ orbitals which act as intermediate states 115. It is also a good approximation for $\mathrm{YBa}_{2} \mathrm{Cu}_{3} \mathrm{O}_{7-x}$ [14].

Eq. (8) can be obtained from the following interlayer hopping Hamiltonian

$$
H_{c}=-\frac{t_{\perp}}{8} \sum_{m, \delta, \delta^{\prime}} D_{\delta} D_{\delta^{\prime}} c_{i+\delta, m}^{\dagger} c_{i+\delta^{\prime}, m+1}+\text { h.c. }
$$

where $m$ is the index of $\mathrm{CuO}_{2}$ planes and $D_{\delta}=1$ or -1 if $\delta= \pm \hat{x}$ or $\pm \hat{y}$. In a vortex system, since the translational symmetry is broken and $\left(k_{x}, k_{y}\right)$ are no longer good quantum numbers, it is more convenient to use this real space form of the c-axis hopping integral. The $c$-axis current operator is now defined by

$$
J_{c}=\frac{i t_{\perp}}{8} \sum_{m, \delta, \delta^{\prime}}\left(D_{\delta} D_{\delta^{\prime}} c_{i+\delta, m}^{\dagger} c_{i+\delta^{\prime}, m+1}-\text { h.c. }\right) .
$$

From the expectation value of $J_{c}$ in the vortex state, we find $v_{k_{z}, n}^{s}$ to be

$$
\left|v_{k_{z}, n}^{s}\right| \sim t_{\perp} V_{n}\left|\sin k_{z}\right|
$$

where

$$
V_{n}=\sum_{i}\left[\left.|| \sum_{\delta} D_{\delta} u_{n}(i+\delta)\right|^{2}+\left|\sum_{\delta} D_{\delta} v_{n}(i+\delta)\right|^{2}\right]
$$

is the $c$-axis velocity factor of the $n^{\prime}$ th vortex state.

Substituting Eqs. (7) and (9) into (6) and integrating out $k_{z}$, we find that the tunneling differential conductance is

$$
g(i, V) \sim \sum_{n} V_{n} \rho(i, n, e V)
$$

If $V_{n}$ does not depend on $n$, such as in an ordinary superconductor, $g(i, V)$ is simply proportional to the LDOS $\rho(i, e V)$. However, as discussed below, in high- $T_{c}$ cuprates $V_{n}$ varies strongly with $n$. In this case $g(i, V)$ is completely different to $\rho(i, \mathrm{eV})$.

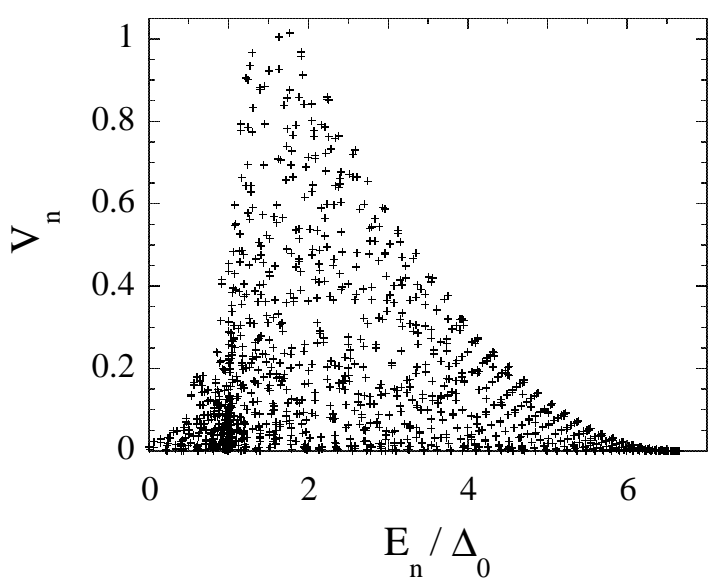

FIG. 2. The $c$-axis velocity factor $V_{n}$ as a function of energy.

Figure 2 shows the energy dependence of $V_{n}$ for the single vortex system discussed previously. The variance of $V_{n}$ is highly non-monotonic. At low energies, $V_{n}$ is very small. It reaches a maximum when $E_{n}$ is slightly above the maximum energy gap $\Delta_{0}$. At high energies, $V_{n}$ drops with increasing energy. The behavior of $V_{n}$ in different energy regions can be understood from the behavior of the expectation value of $\left(\cos k_{x}-\cos k_{y}\right)^{2}$ in each vortex eigenstate. At low energies, $V_{n}$ is small since it arises mainly from excitations around the gap nodes. In this energy region, $V_{n}$ has roughly the same energy dependence as $\left(\cos k_{x}-\cos k_{y}\right)^{2}$ and its local maximum increases approximately with $E^{2}$. $V_{n}$ becomes large when $E_{n}$ is approximately equal to $\Delta_{0}$. This is because the maximum gap corresponds to the contribution of the quasiparticle around $(\pi, 0)$ where $\left(\cos k_{x}-\cos k_{y}\right)^{2}$ is maximum. The high energy eigenstates are mainly from the excitations around $(0,0)$ and $( \pm \pi, \pm \pi) . \quad V_{n}$ drops at high energies since $\left(\cos k_{x}-\cos k_{x}\right)^{2}=4 \sin ^{2}\left(k_{x}+k_{y}\right) / 2 \sin ^{2}\left(k_{x}-k_{y}\right) / 2$ and $\sin ^{2}\left(k_{x}+k_{y}\right) / 2$ is zero at $(0,0)$ and $( \pm \pi, \pm \pi)$.

Figure 3 shows the tunneling differential conductance for the system studied above. The conductance at $(10,10)$ behaves in qualitatively the same way as for the LDOS, but its coherent peak at the gap edge is suppressed. At site $(0,0)$ (the core center), the conductance differs completely from the LDOS. In particular, three distinct features appear in the tunneling conductance. (1) The $c$-axis velocity $V_{n}$ suppresses completely the zero energy peak of LDOS and leads to a small tunneling conductance at zero bias. The zero bias conductance is finite in the figure. This is due to the broadening of the spectrum by the parameter $\gamma$. In an infinite lattice system where $\gamma$ can be asymptotically set to zero, the value of the zero bias conductance should become zero since the tunneling matrix vanishes at the gap nodes. (2) There are two weak peaks at low bias. The positions of these weak peaks depend on $\Delta_{0}$. For the case shown in Figure 2 , they are located at $V \sim \pm 0.25 \Delta_{0}$. These peaks result 
from the competition between the decreasing density of states and the increasing velocity factor (on average) at low energies. Unlike the irregular oscillations caused by the finite size effects, the positions of these peaks do not change as the lattice size is increased. This shows that these weak peaks are not due to the finite size effects. The peak value is very small compared with the conductance at the gap edge $|V| \sim \Delta_{0}$, but it slightly increases with increasing lattice size. The existence of these low bias peaks with the absence of the zero bias peak is reminiscent of the tunneling spectrum of a conventional $s$-wave superconductor, although no vortex core bound states exist in this case. (3) There is no coherent peak at the gap edge. This is different than in a conventional $s$-wave superconductor. All these three features agree well with the experimental observations [1] 3 .

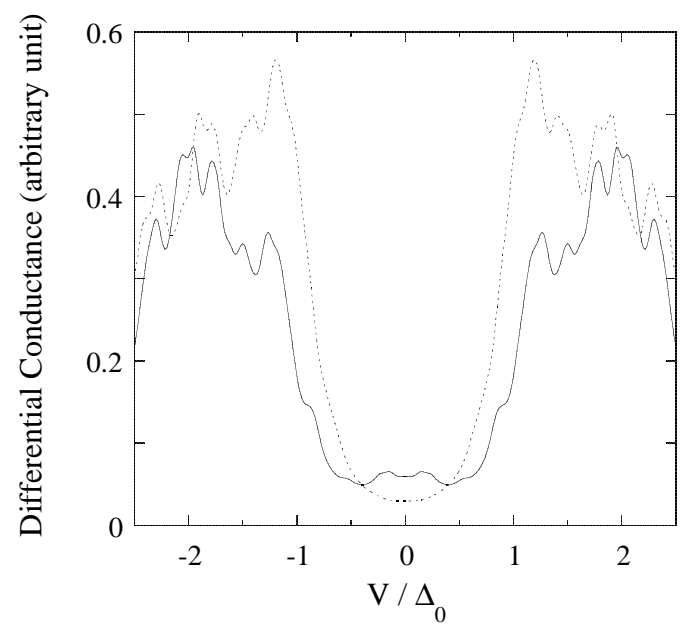

FIG. 3. Tunneling differential conductance at the core center at site $(0,0)$ (solid line) and at site $(10,10)$ (dotted line) for the same system as shown in Figure 1.

In the above discussion, particle-hole symmetry has been implicitly assumed since the chemical potential $\mu$ has been set to zero. For a particle-hole asymmetric system $(\mu \neq 0)$, we find that the differential conductance becomes asymmetric with respect to zero bias. However, the other features of the tunneling spectrum seen in the particle-hole symmetric system are unchanged. Therefore, we believe the asymmetry of the STS observed in the experiments is mainly due to the breaking of particlehole symmetry [1] 3 .

In conclusion, we have presented a theoretical analysis of the vortex tunneling spectra of high- $T_{c}$ superconductors. Our work indicates that it is important to include the anisotropy of the c-axis hopping integral in the analysis of the STS in high- $T_{c}$ oxides. The tunneling matrix elements parallel to $\mathrm{CuO}_{2}$ planes do not have the same anisotropy as the $c$-axis case. Thus STS for a vortex perpendicular to the $c$-axis is expected to behave very differently. In particular, the zero energy peak of the LDOS at the core center should appear in the STS in this case.
Thus by measuring the STS parallel to $\mathrm{CuO}_{2}$ planes, we can obtain a better understanding of the $c$-axis tunneling experiments.

We wish to thank S. Pan and C. Panagopoulos for useful discussions. This work was supported in part by the National Natural Science Foundation of China and by the Special funds for Major State Basic Research Projects of China.

[1] I. Maggio-Aprile, Ch. Renner, A. Erb, E. Waler and O.Fischer, Phys. Rev. Lett. 75, 2754(1995).

[2] Ch. Renner, B. Revaz, K. Kadowaki, I. Maggio-Aprile and O. Fischer, Phys. Rev. Lett. 80, 3606(1998).

[3] S. H. Pan, E. W. Hudson, A. K. Gupta, K. W. Ng, H. Eisaki S. Uchida and J. C. Davis, Condmat/0005484.

[4] P. I. Soininen, C. Kallin, and A. J. Berlinshy, Phys. Rev. B 50, 13833 (1994)

[5] Y. Wang, A. H. MacDonald, Phys. Rev. B 52, R3876(1995).

[6] Y. Morita, M. Kohmoto, and K. Maki, Phys. Rev. Lett. 78, 4841 (1997).

[7] D. P. Arovas, A. J. Berlinsky, C. Kallin, and S.-C. Zhang, Phys. Rev. Lett. 79, 2871 (1997)

[8] M. Franz and Z. Tesanovic, Phys. Rev. Lett. 80, 4763(1998).

[9] K. Yasui and T. Kita, Phys. Rev. Lett. 83, 4168(1999).

[10] B. M. Andersen, H. Bruus and P. Hedegard, Phys. Rev. B 61, 6298 (2000)

[11] M. Ichioka, N. Hayashi, N. Enomoto, and K. Machida, Phys. Rev. B 53, 15316 (1996).

[12] M. Franz and Z. Tesanovic, cond-mat/0002137.

[13] D.-H. Lee, Phys. Rev. Lett. 84, 2694 (2000).

[14] O. K. Andersen, O. Jepsen, A. I. Liechtenstein, and I. I. Mazin, Phys. Rev. B 49, 4145 (1994).

[15] T. Xiang and J. M. Wheatley, Phys. Rev. Lett. 77, 4632 (1997); T. Xiang, C. Panagopoulos and J. R. Cooper, Int. J. Mod. Phys. B 12, 1007 (1998).

[16] S. Chakravarty, A. Sudbo, P. W. Anderson, S. Strong, Science 261, 331 (1993).

[17] C. Panagopoulos, J. R. Cooper, T. Xiang, G. B. Peacock, I. Gameson, P. P. Edwards, Phys. Rev. Lett. 79, 2320 (1997); M. B. Gaifullin, Y. Matsuda, N. Chikumoto, J. Shimovama, K. Kishio, and R. Yoshizaki, Phys. Rev. Lett. 83, 3928 (1999).

[18] A. Hosseni, S. Kamal, D. A. Bonn, R. Liang, and W. N. Hardy, Phys. Rev. Lett. 81, 1298 (1998); T. Xiang and W. N. Hardy, cond-mat/0001443.

[19] M. Franz and Z. Tesanovic, Phys. Rev. B 60, 3581 (1999).

[20] C. Caroli, P. G. de Gennes, and J. Matricon, Phys. Lett. 9, 307 (1964).

[21] P. G. de Gennes, Superconductivity of Metals and Alloys (Addison-Wesley, Reading, MA, 1989)

[22] E. L. Wolf, "Principles of Electron Tunneling Spectroscopy" (Oxford University Press, United Kingdom, 1985). 